\title{
Algorithm and Data Research of Computer Aided Course
}

\section{Arrangement System}

\author{
Weili Chu,Yanmei Meng
}

Qingdao Huanghai University

\begin{abstract}
Keywords: Computer aided system, Course arrangement, Algorithm optimization
\end{abstract}
\begin{abstract}
In course arrangement, the factors, such as time, courses, teaching areas, classrooms, faculties, classes, teachers, and so on need to be taken into consideration. The conflicts in any period of time in the teacher, the teaching, the class of the teaching, the classroom occupation will be avoided in the course arrangement after being optimized and it is reasonable in measuring the school timetable in a comprehensive way. This thesis makes analysis on the demand of arranging courses and expounds the whole organization and implementation plan of the system. According to the requirements of the artificial course arrangement, an automatic course arrangement algorithm based on priority is designed. Experimental results show that this method is effective and can solve the outstanding problems in timetable arrangement.
\end{abstract}

\section{Introduction}

Course arrangement is an important, complex and basic work in school teaching management. The essence of it is to arrange a proper set of teaching time and space for the courses set by the school, so that the whole teaching can be carried out in a planned and order way. Because of the characteristics such as: large scale, complex constraint (requirement) and constant changing nature, the timetable problem becomes one of the interesting subjects. Higher vocational education is different from the traditional discipline education in the aspects of training objective, school running mode and teaching method. It should follow the principles of "Necessity" and "Sufficiency"[1], and "Theory serves practice" by focusing on training students' practical ability, and cultivating higher technology application-oriented talents for production, construction, management and service. The characteristics of training objectives and training methods determine that the educational administration of higher vocational education faces many new difficulties, and among them[2], the problems such as arranging courses, adjusting classes, checking teaching progress, quality and communicating the teaching information of both sides are particularly prominent[3]. It plays an important role in maintaining the normal teaching order of higher vocational colleges and improving the teaching effect on how to utilize the limited teachers and classroom resources to arrange a reasonable curriculum. Higher vocational colleges should be based on their own actual situations and develop their own timetable systems.

According to the characteristics of the teaching system in higher vocational colleges, in the thesis, the mathematical model of course arrangement problem is analyzed and an automatic course arrangement algorithm based on priority is designed. The algorithm reduces the complexity of the course arrangement by dividing the equivalence class and calculating 
the priority so as to obtain more satisfactory results of course arrangement at a faster speed. At the same time, in the process of designing the algorithm, it also pays great attention to the solution of the conflict.

\section{Requirement analysis}

The main content of this thesis is to use the computer to realize the functions of the arrangement of the timetable, the processing of basic data, the inquiry of the class schedule and the output of the report forms. As a complete application system, it mainly involves the following requirements:

Processing of basic data

The course arrangement involves much basic data, which includes the faculties, teachers, students, classrooms, courses and other information. We should be able to flexibly add, delete, and modify the basic information according to the actual situation.

Management of course arrangement

Course arrangement is not arbitrary and it is a combinatorial programming problem of four factors: time, teacher, student and classroom. In order to achieve the best teaching effect, certain requirements should be followed and these requirements mainly include:

(1)Try to arrange the best time for the courses offered for the scheduled courses;

(2)The course has a certain time interval over the course that has several times a week;

(3)Courses involving a wide range and longer hours, such as public course and so on, should be given priority;

(4) The relatively fixed classrooms should be chosen as much as possible for the same teacher and the same class;

(5)In cases, special requests are put up with by teachers, students, courses and so on, they should be dealt with according to the specific circumstances.For example: teachers who need to take children to school are not scheduled for the first or second class in the morning; older teachers are not suitable to be scheduled for the first or second class in the morning; teachers and classes with out-of-school guidance or teaching practice should finish the class plan of this semester within the appointed time of the semester; a teacher who has temporarily added teaching tasks should deal with the conflicts between the new class hours and the original timetable. In addition, some special courses, such as single biweekly courses and irregular weekly courses, also need to be considered and be arranged in reasonable. Original course arrangement results by computer can also be modified by human interaction.

Dealing with the course arrangement results

After that the computer has finished arranging the course, all kinds of inquiries can be done on the results and tables with multiform can be output according to the situation in order to facilitate the management by academic staff. For example: course arrangement for a class, course arrangement for a teacher and usage of a classroom can be inquired.

Making full use of the advantages of the network

The advantages of the network should be made full use of and computer distributed course arrangement should be put into practice. Computer course arrangement requires the computer to check the various conflicts within the school, therefore, most of the early computer course 
arrangement systems took centralized course arrangement, which was usually completed by the dean office. But now all schools are expanding their enrollment, which leads to that there are too many students and too many classes, and more basic data needs to be input in course arrangement, so it is very impractical to make the task of course arrangement completed by a department. The only way for implementing computer course arrangement is to decompose the task of course arrangement, break up the whole into parts and carry out distributed computer course arrangement. Now most colleges and universities have set up campus network, which makes it possible to put distributed computer course arrangement into practice. The basic information such as courses, teachers, students, classes and classrooms can not only be input temporarily, but also can be obtained from other teaching management systems or campus networks to reduce data entry workload.

\section{Algorithmic strategy of course arrangement system}

There are many constraints to the timetable problem, which can be summarized as following: $m$ is the total number of classrooms available for the whole school, $n$ is the number of classes in the whole school, $\mathrm{p}$ is the number of courses for all classes in the school, $q$ is the sum of the time periods for each classroom within a week, $t$ is the total number of teachers, $\mathrm{w}$ is the number of time periods available within a week,C1, C2, C3, and C4 are penalized values for different conflicts that may arise from the timetable. For a given class $\mathrm{u}$, the course timetable is located in the Y matrix and lu-lu'line.

1. The number of weekly hours for a certain course $\mathrm{i}$ is a definite number bi(Referring to formula 1):

$$
2 * \sum_{j=0}^{\mathrm{q}-1} y_{i j}=b_{i} \quad \mathrm{i} \in[0, \mathrm{p}-1]
$$

2. One teacher can only take one course at a period of time(Referring to formula 2):

$$
\mathrm{X}_{r s} \leq 1 \quad r \in[0, t-1], s \in[0, w-1]
$$

3. A certain period of time in a classroom can only be taken up by one course(Referring to formula 3):

$$
\sum_{i=0}^{\mathrm{q}-1} y_{i j} \leq 1 \quad \mathrm{j} \in[0, q-1]
$$

4. A class can only be arranged in one classroom for a certain period of time(Referring to formula 4):

$$
\sum_{a=l u}^{h i^{\prime}} \sum_{b=0}^{m-1} y_{a\left(b^{*} w, v\right)} \leq 1 \quad V \in[0, w-1]
$$

In view of the above constraints, mathematical model for timetable problem is given(Referring to formula 5):

$$
g=c_{1} \sum_{j=0}^{q-1} \Phi_{1}+c_{2} \sum_{r=0}^{t-1} \sum_{\lambda=0}^{w-1} \Phi_{2}(r, s)+c_{3} \sum_{u=0}^{n-1} \sum_{v=0}^{w-1} \Phi_{3}(u, v)+c_{4} \sum_{i=0}^{p-1} \sum_{j=1}^{q-1} y_{i j} * y_{i(j-1)}
$$




\section{Design and implementation of time arrangement}

After finishing the pre-treatment work, each subclass can be conducted course arrangement. In the course arrangement of each subclass, we combine priority, dividing conquering and greedy method. First, the priority of each course in the subclass is calculated, and the course with high priority can be given priority scheduling. Then, according to the thought of dividing conquering method, the whole course arrangement process is divided into two stages: time allocation and classroom allocation. Finally, based on the algorithm idea of greedy method, in the time allocation, the unit with the best class effect is selected in the time units that have not yet been allocated, that is, the time model for the best class effect is selected. The concrete process is as following:

Setting priority

Arranging order of the course arrangement is directly related to the success of the course arrangement and the rationality of the timetable, therefore, the determination of setting priority plays an important role in the course arrangement process.

The following example, as shown in Figure 1,can be examined. It can be assumed that class B1 and class B2 are individually take courses $\mathrm{K} 1$ and courses $\mathrm{K} 2$ and B1 and B2 want to take courses together. Supposing that the priorities of K1 and K2 are higher than the priority of K3, then $\mathrm{K} 1$ and $\mathrm{K} 2$ are scheduled prior to K3. If $\mathrm{K} 1$ is scheduled in the time unit $\mathrm{H} 1, \mathrm{~K} 2$ is arranged in the time unit $\mathrm{H} 2$, and the $\mathrm{K} 3$ is available at $\mathrm{HI}+\mathrm{H} 2$, then the scheduling of $\mathrm{K} 3$ is bound to fail.

\begin{tabular}{|c|c|c|c|}
\hline & K1 & K2 & K3 \\
\hline H1 & & & \\
\hline H2 & & & \\
\hline
\end{tabular}

Figure 1 Conflicting constraints for K1、K2、K3

In fact, as long as the priority of these three courses is adjusted, that is to say, the priority of the course scheduling is correspondingly adjusted, the deadlock problem of $\mathrm{K} 3$ can be avoided. The method is: adjusting the priority of the K3 to make the K3 prioritize, and then adjusting the $\mathrm{K} 1$ and $\mathrm{K} 2$, as shown in Figure 2 and Figure 3, and the deadlock problem cam be solved.

\begin{tabular}{|c|c|c|c|}
\hline & K1 & K2 & K3 \\
\hline H1 & & & \\
\hline H2 & & & \\
\hline
\end{tabular}

Figure 2 Course arrangement result A after changing course arrangement priority 


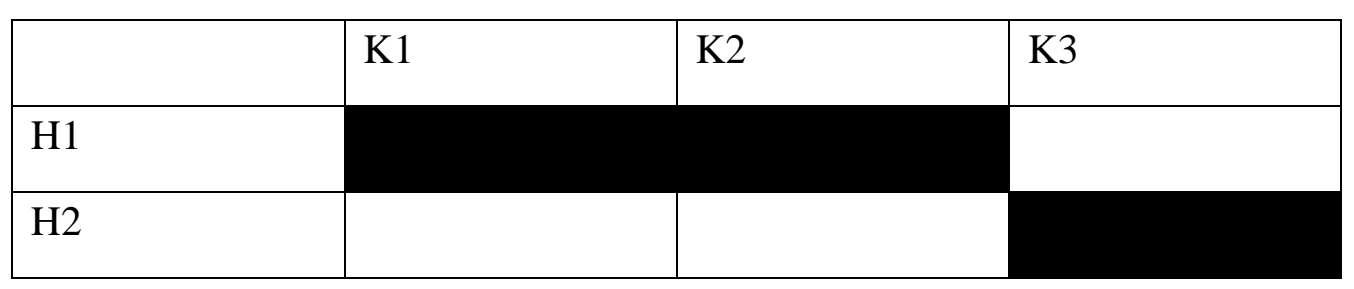

Figure 3Course arrangement result B after changing course arrangement priority

\section{Conclusion}

Course timetable arrangement is one of the typical problems of combination scheduling and there is a high demand for both rationality and reliability. Its special complexity has attracted a large number of researchers at home and abroad to study it, but so far, there is no computer scheduling system that users generally response well.

On the basis of analyzing the existing methods of course arrangement, this thesis designs and realizes the simple algorithm of computer scheduling system based on the characteristics of higher vocational education and puts up with a new automatic scheduling algorithm based on priority. By using partition equivalence class and computing priority method and so on, the algorithm hierarchically processes the whole problem so that the contradictions are scattered among the sub problems. By hierarchical management[4], the aims of reducing algorithmic complexity and decreasing deadlock can be reached, by which relatively fast processing speed and satisfactory processing results can be obtained.

Due to the characteristics of large scale, complex constraints and nature changing of timetable problems, In this thesis, there are still some works to be done to improve the course arrangement algorithm, for instance, the management of irregular weekly course and the manual adjustment of results of computer timetabling and so on. Although there are still some shortcomings in the algorithm, a new idea has been put forward in the study of the timetable problem, which has reached the aim of simplifying the algorithm.

\section{References}

[1] Esteva A, Kuprel B, Novoa R A, et al. Dermatologist-level classification of skin cancer with deep neural networks[J]. Nature, 2017, 542(7639): 115-118.

[2] Lin J, Yang B, Xiao Z, et al. The Design of the Household Appliances Remote Control System Based on GSM[J]. Journal of Applied Science and Engineering Innovation, 2016, 3(1): 26-29.

[3] Xie D, Zhang Y, Xiao J, et al. Training Creative Talents for Graduate Students of the Agriculture Information Field in China[J]. Journal of Applied Science and Engineering Innovation, 2016, 3(2): 66-68.

[4] Mizera-Pietraszko, Jolanta, and Pit Pichappan, eds. Lecture Notes in Real-Time Intelligent Systems. Vol. 613. Springer, 2017. 